\title{
Comunicação e Interação no Ensino Através do Uso de Redes Sociais Virtuais
}

Wellington Tavares - CEPEAD/UFMG e CEAD/UFOP - wellington@ cead.ufop.br Helton Cristian de Paula - CEPEAD/UFMG e CEAD/UFOP - helton@ cead.ufop.br Ana Paula Paes de Paula - CEPEAD/UFMG - appp.ufmg@gmail.com

Resumo: Este estudo apresenta uma discussão sobre o uso de redes sociais virtuais de forma a complementar as atividades realizadas em sala de aula no ensino presencial, não demonstrando relações diretas com a utilização de tecnologias informacionais e comunicacionais na modalidade de ensino à distância. No decorrer do estudo depara-se com uma discussão teórica que envolve vários aspectos e informações sobre as redes sociais virtuais e suas possibilidades para os usuários, dando enfoque especial ao seu uso na educação. Na sequência é apresentado um caso de ensino no qual a utilização de um grupo virtual no Facebook foi utilizado, bem como dos resultados que se mostraram muito satisfatórios, já que o uso trouxe melhorias no aprendizado dos alunos, conforme a própria visão e avaliação destes envolvidos que são apresentadas neste caso de ensino. Palavras-chave: comunicação; interação; redes sociais virtuais; ensino.

\section{Communication and Interaction in Learning Through the Use of Virtual Social Networks}

Abstract: This study presents a discussion about the use of virtual social networks to complement the activities carried out in the in traditional face to face classroom, while not demonstrating direct relations with the use of information and communication technologies in distance learning modalities. In this study the authors share a theoretical discussion involving various aspects and information about virtual social networks and the possibilities for users, giving a special focus to its use in education. Following a report using a case study that used virtual Facebook group, where the results were satisfactory. Data from this study support the supposition that the use of the virtual environment brought improvements in student learning.

Key-words: communication, interaction, virtual social networks; teaching. 


\section{Introdução}

A educação, tal quais inúmeros outros setores da sociedade, tem sofrido grandes impactos e transformações a partir do avanço da tecnologia e dos meios de comunicação disponíveis na atualidade. Desde o surgimento e popularização dos computadores e da internet, a forma de lidar com os processos de ensino e aprendizagem vêm tomando novas formas, desafiando os professores a se adaptarem ao uso de tecnologias como forma de acompanhar as demandas de alunos e sua própria forma de trabalho.

A popularização das redes sociais, como o Facebook, veio reforçar o contexto das novas formas de relações e comunicação, o que se alia recentemente à intensa propagação de novos instrumentos de comunicação. Além dos softwares que dão base para as redes sociais virtuais, o crescente uso de smartphones e tablets tem permitido o acesso instantâneo à internet, o que dinamiza ainda mais a comunicação e interações das pessoas.

Este contexto também influencia o campo da educação, pois há a incorporação de novas formas de se realizar os processos de ensino, mesmo que tal mudança ocorra como forma de complementar as relações de sala de aula do Ensino Presencial e não como Ensino à Distância. Com as novas nuances e desafios pelas quais passam os envolvidos no campo da educação muitos questionamentos surgem. Neste estudo, o enfoque dado parte da seguinte questão de pesquisa: Quais as possibilidades presentes no processo de comunicação e interação de professor e alunos a partir da utilização de redes sociais virtuais?

Para responder tal questionamento, este estudo objetiva analisar e discutir o processo de comunicação e interação de professor e alunos através do uso de redes sociais virtuais em um caso de ensino. Além de uma breve fundamentação teórica sobre as redes sociais virtuais e sua aplicação em processos de ensino, este estudo apresenta o caso de um grupo virtual inserido no Facebook como forma de expandir as possibilidades de comunicação e interação entre alunos e professores.

Como forma de organizar a discussão proposta e alcançar o objetivo pretendido, este estudo está organizado em seções. Na primeira seção são apresentados conceitos, histórico, características e formas de utilização de redes sociais virtuais, dando ênfase na utilização na educação. Na terceira seção apresenta-se uma breve metodologia que baseou o caso de ensino apresentado e na quarta seção este caso é discutido. Nesta seção são apresentadas duas análises do caso: quantitativa e qualitativa. Por fim, na quinta seção apresentam-se as considerações finais deste estudo.

\section{Redes Sociais Virtuais e a Educação}

A partir do conceito de redes sociais desenvolvido na Sociologia e da ampla gama de trabalhos, que buscam esclarecer o modo como se dão os processos de formação e desenvolvimento destas (CASTELLS, 1999; MARTELETO, 2001; WELLMAN, 2002; CRUZ e QUANDT, 2007), novas concepções de interação tem sido discutidas. Tais redes, conhecidas também como "redes sociais na internet" se referem às novas formas de sociabilidade e de relacionamentos sociais a partir de ambientes virtuais - ciberespaço -, diferentes da dinâmica e dos objetivos das redes desenvolvidas nos ambientes reais. Apesar das diferenças claras entre redes virtuais e reais, estas últimas podem se servir das primeiras como um ambiente adicional e complementar (AGUIAR, 2007).

De modo geral, obviamente guardadas algumas exceções, os indivíduos demonstram uma necessidade de se integrar a grupos sociais específicos, seja objetivando interesses comuns ou mesmo pela identificação com estes grupos e os indivíduos que os formam. A identificação e inserção em redes sociais, em especial por 
meio do ambiente virtual proporcionado pela internet, permitem a criação de espaços e condições de comunicação (CASTELLS, 1999).

A redução das possibilidades de encontros reais tem fortalecido e desenvolvido as configurações de um novo conceito de tempo-espaço no qual as relações ocorrem e se estabelecem independentemente do tipo e das motivações para as relações, já que estas permitem criar inclusive laços emotivos (RHEINGOLD, 1996). Apesar de as redes assumirem possibilidades positivas como amizade e relações de tolerância, também possibilitam comportamentos negativos como o ódio e formas de violência, o que supõe a não utilização de normas de etiquetas no ambiente virtual, conceituada como 'netiqueta' (PRIMO, 1997). Na 'netiqueta' estão incluídas regras relacionadas aos comportamentos esperados dos usuários. Entre estas regras figuram principalmente as relacionadas aos comportamentos aguardados e como forma de disciplinar $\mathrm{o}$ comportamento dos indivíduos e do grupo (SILVA et al., 2008).

Uma rede social virtual normalmente é estruturada de acordo com os conhecimentos e interesses mútuos de seus integrantes em um processo pautado por trocas e cooperação, sem necessitar exclusivamente de uma proximidade geográfica e institucional entre os integrantes (LÉVY, 2000), já que os meios de comunicação suprem a demanda das redes sociais por compartilhamento de informações e comunicação colaborativa (WELLMAN, 2002). Para Lemos e Lévy (2010, p. 101) a formação de redes sociais on-line é um meio de se "fazer sociedade". Wellman e Berkowitz (1988) defendem que as redes sociais sustentam uma estrutura de rede na sociedade e não pode ser classificadas como meras metáforas para compreender agrupamentos de indivíduos.

As redes sociais virtuais são possíveis a partir dos ciberespaços originados do desenvolvimento das Tecnologias de Informação e Comunicação (TICs), da Web 2.0 e de recursos computacionais disponíveis, destacando-se os e-mails e seus grupos, chats e, em especial, os softwares sociais. Vários agentes e setores na sociedade têm se servido destas redes, tais como setores públicos e de negócios de diferentes naturezas, movimentos sociais e culturais, e, em especial, a educação e os sistemas de ensino (MACHADO; TIJIBOY, 2005). Tais redes não apenas possibilitam a interação, encontro de pessoas e desenvolvimento de relações sociais, mas se destacam por permitirem a criação de espaços de aprendizado coletivo, bem como de trocas de conhecimento de forma coletiva (RHEINGOLD, 1996; MACHADO e TIJIBOY, 2005).

$\mathrm{Na}$ busca por um histórico da criação de redes sociais virtuais, depara-se com o exemplo de uma apresenta o exemplo da Comunidade WELL (Whole Earth Eletronic Link), a qual foi criada e desenvolvida em São Francisco nos EUA no ano de 1985. Esta primeira rede se caracterizou como um sistema de teleconferência em formato de texto realizado por computadores que permitiam uma forma de reprodução do mundo real das pessoas, envolvendo emoções e cooperação entre os membros e criando um ambiente de interação social (RHEIGOLD, 1996).

O conceito e aplicabilidade de software social, ou mesmo mídia social, foi desenvolvido com vistas a suportar a interação de grupos, ainda que a interação seja realizada de no modo off-line (BOYD, 2007). Um breve histórico do desenvolvimento dos softwares sociais descritos por Tavares e Paula (2013) com base em Boyd e Ellison (2007) demonstra que entre estes softwares

o precursor é o SixDegrees.com, lançado no ano de 1997. Após isto, até meados de 2001, uma série de sites surgiu com diferentes possibilidades para formar as redes sociais, conforme anos de surgimento e nomes a seguir: 1999 - LiveJournal, AsianAvenue, BlackPlanet; 2000 - MiGente; 2001- Cyworld e Ryze; 2002 - Fotolog e Friendster; 2003 - LinkedIn, MySpace, Tribe.net, Last.FM e Hi5; 2004 - Orkut, Flickr e Piczo; 2005 - Yahoo! 360 e YouTube, e; 2006 - Windows 
Live Spaces, Twitter e Facebook. Apesar de o Facebook ter se popularizado a partir de 2006, ele já podia ser utilizado em 2004 apenas por um grupo restrito em Harvard, além de uma versão para redes corporativas lançada no início de 2006. Atualmente, outra rede que tem crescido em popularidade e membros é a Google+, lançada pela Google em meados de junho de 2011 para fazer frente ao crescimento do Facebook e, em especial, diante da queda no número de usuários do Orkut, sua outra rede social. Mais recentemente, outra rede social entrou neste cenário no final de 2012, a Socl, desenvolvida pela Microsoft. (TAVARES e PAULA, 2013, p. 4-5).

Conforme quadro conceitual apresentado em relação às redes sociais virtuais tornou-se necessário analisar de forma prática como as redes sociais podem permitir a criação de um espaço complementar para o processo de aprendizagem dos alunos de forma a criar condições de interação e comunicação entre estes e o professor. Para alcançar tal objetivo, o estudo enfocou a rede social virtual Facebook como ambiente no qual se estabeleceu um grupo com fins educacionais, conforme se verá adiante no caso apresentado.

Cheung et al. (2011) argumentam que a maioria das pessoas usa o Facebook para conseguir comunicação e conexão instantânea com os amigos. Este serviço tem várias funcionalidades comuns a outros serviços de redes sociais, mas apresenta ainda uma página denominada feed de notícias, onde estão concentradas as atualizações feitas por dois grupos; os amigos, usuários adicionados pelo utilizador como amigos; e as fan pages, páginas nas quais o usuário se cadastrou como fã/seguidor. Estas ferramentas permitem o acesso a várias informações com um único acesso e permite ao usuário definir sobre quais assuntos e de quais pessoas deseja receber atualizações.

Minhoto (2012) defende que a utilização do Facebook como apoio ao ensino presencial, com as suas ferramentas de interação e colaboração, permite expandir a sala de aula para um contexto muito familiar aos alunos, menos rígido do que uma plataforma de aprendizagem (como, por exemplo, a plataforma Moodle). O Facebook se mostra como uma possibilidade com maior interatividade e de participação. A mesma autora argumenta que a familiaridade dos alunos com o ambiente deste software social diminui a curva de aprendizagem das ferramentas, o que facilita a utilização e estimula a participação. A utilização de uma plataforma onde o período de aprendizagem é mais longo constitui, frequentemente, fator de desmotivação de uma utilização rotineira.

Um estudo conduzido por Selwyn (2009) sobre o uso educacional do Facebook concluiu que este pode ser um meio de suporte da comunicação entre os alunos e entre alunos e professores. Llorens e Capdeferro (2011) descrevem a aprendizagem colaborativa como principal potencialidade pedagógica do Facebook, que favorece a cultura de comunidade virtual e da aprendizagem social. Esta cultura fundamenta-se em valores que surgem nos usuários ao interagirem sobre um determinado tema ou com um objetivo em comum, gerando sentimentos de pertencimento e uma identidade social. Ao mesmo tempo, suporta ainda abordagens inovadoras da aprendizagem, pois são inúmeras as possibilidades para permitir a construção do conhecimento e desenvolver competências.

\section{Metodologia}

Este trabalho se apresenta como um estudo de caso de ensino, no qual são apresentadas as experiências e discussões sobre um caso de utilização de redes sociais virtuais como complemento às atividades desenvolvidas em sala de aula. A coleta de dados primários se deu a partir de um questionário semiestruturado abrigado em um site (Google Docs), o qual foi disponibilizado para os alunos participantes por meio de um link no próprio grupo virtual que fizeram parte no decorrer da disciplina. 
Para subsidiar a análise de dados do ponto de vista quantitativo, optou-se por utilizar a estatística descritiva. A base de dados obtida através da aplicação do questionário da pesquisa foi tratada levando em consideração esta técnica, e os dados obtidos classificaram todas as respostas em termos percentuais de acordo com a quantidade de respondentes e as opções de cada uma das questões. Os dados foram agrupados em uma tabela, conforme será apresentado no tópico de análise de dados quantitativos. Na análise qualitativa levaram-se em consideração as respostas descritivas dos alunos, tendo sido organizadas em um quadro a partir de categorias estabelecidas durante a análise e que representassem as opiniões dos respondentes em relação aos processos de comunicação e interação, bem como os resultados destes processos para o próprio processo de ensino da disciplina em questão.

\section{Apresentação do Caso e Discussão}

O caso de ensino no qual se baseia este estudo ocorreu a partir de uma disciplina presencial ofertada por um dos autores, que é professor da Universidade Federal de Ouro Preto (UFOP). A disciplina em questão denomina-se "Administração e Organização I", a qual se baseia em apresentar fundamentos básicos das Teorias de Administração. Tal disciplina foi ofertada no segundo semestre letivo de 2012 (2012/2) para três turmas heterogêneas formadas por alunos de vários cursos de diferentes áreas de Engenharia e também da Museologia, da Ciência da Computação, Química, entre outros, formando um total de 169 alunos - sendo três turmas de 58, 58 e 53 alunos cada.

A importância do caso de ensino em questão, e que este estudo busca ressaltar e apresentar, está na utilização de um grupo no Facebook como forma de estender e complementar a interação e comunicação com os alunos além da sala de aula. Desta forma, dos 169 alunos mencionados, 156 aderiram ao grupo desde o início da disciplina até o final da mesma. $\mathrm{O}$ grupo virtual foi utilizado pelo professor e pelos alunos como forma de disponibilizar informações e criar espaço para comunicação professor-alunos, alunos-professor e aluno-alunos. Além disso, ao final da disciplina o próprio grupo virtual foi útil para apresentar o questionário aos alunos e requerer a participação dos mesmos neste estudo.

O material publicado durante o semestre letivo se divide em dois grupos: a) publicações do professor, composta por: slides de aula; atividades para entrega em sala de aula; links de vídeos e reportagens com material complementar; esclarecimento de questões relacionadas às atividades; disponibilização de planilhas de notas parciais e finais para acompanhamento dos alunos; avisos gerais, e; b) publicações de alunos, composta por: dúvidas e demandas de esclarecimentos; comunicação com outros alunos para formação de grupos para realizar atividades; postagens de vídeos e links relacionados à disciplina como forma de sugestões de conteúdo.

O grupo virtual no software social, com o mesmo nome da disciplina, apresentou a seguinte definição "Este grupo se destina à publicação de materiais didáticos da disciplina PRO243 - Organização e Administração 1 (UFOP), bem como demais informações relevantes que apresentem relação com a disciplina.", o que foi necessário para definir o tipo de participação esperada dos alunos e do professor, conforme discutido por Silva et al. (2008) sobre 'netiqueta'.

Dos 156 alunos participantes do grupo, 54 responderam o questionário que dá base para as discussões que virão na sequência. A amostra contou com 54 respondentes, dentre os quais $72 \%$ do sexo masculino e $28 \%$ do sexo feminino. Aproximadamente $74 \%$ dos respondentes declararam sua faixa etária entre 21 e 24 anos de idade, $18 \%$ declararam possuir entre 25 e 28 anos de idade, e somente $4 \%$ possuem 20 anos ou 
menos ou acima de 28 anos de idade. A maioria dos alunos, $83 \%$ estão matriculados no curso de Engenharia em uma de suas habilitações, e os outros $17 \%$ dos respondentes estão matriculados no curso de Química ou Museologia.

\subsection{Análise Quantitativa quanto à utilização do Facebook}

Através da coleta de dados primários obtidos por meio da aplicação de um questionário online, foi analisado o processo de interação e comunicação entre alunos e professor. As questões propostas no questionário se dividiram em dois grupos: o processo de comunicação e o processo de interação. Cabe destacar que no processo de interação foi avaliado não só a relação entre alunos com o professor, mas também o processo de interação entre os alunos. A partir de uma série de questionamentos buscando identificar a percepção da importância do grupo virtual no Facebook como complementos da disciplina foram feitas perguntas com as seguintes possibilidades de resposta: muito importante - importante - indiferente - desnecessária - muito desnecessária. Foram encontradas as seguintes percepções dos alunos:

\begin{tabular}{|c|c|c|c|}
\hline Questão & $\begin{array}{c}\text { Muito } \\
\text { Importante }\end{array}$ & Importante & Indiferente \\
\hline Disponibilização de Materiais Didáticos & $85 \%$ & $13 \%$ & $2 \%$ \\
\hline Disponibilização de Conteúdos Complementares & $50 \%$ & $50 \%$ & \\
\hline Disponibilização de Planilhas de Notas & $80 \%$ & $17 \%$ & $4 \%$ \\
\hline Disponibilização de informações sobre atividades & $76 \%$ & $22 \%$ & $2 \%$ \\
\hline $\begin{array}{l}\text { Iniciativa de interagir com os alunos por meio da } \\
\text { rede }\end{array}$ & $80 \%$ & $19 \%$ & $2 \%$ \\
\hline Espaço de interação com o professor & $76 \%$ & $24 \%$ & \\
\hline Espaço de interação com os colegas & $72 \%$ & $24 \%$ & $4 \%$ \\
\hline
\end{tabular}

Tabela 1 - Processo de interação e comunicação com o uso de rede social virtual

Fonte: Elaborada com base nos dados da pesquisa

Ao analisar os dados acima, observa-se que nas questões destinadas a avaliar a disponibilização de informações, a grande maioria dos alunos, $85 \%$, considerou muito importante o uso da rede social virtual para a disponibilização de materiais didáticos. Por outro lado, somente $50 \%$ deles consideraram como muito importante a disponibilização de conteúdo complementar. Este resultado denota que apesar de todos os alunos consideraram muito importante ou importante a disponibilização de materiais complementares, a maioria deles está interessada no uso do espaço para reunir os materiais didáticos base da disciplina, normalmente aqueles que servem de base para $o$ processo de atribuição de notas e avaliação.

Nas questões destinadas a analisar a interação por meio da rede social virtual, novamente a maioria dos alunos considerou o processo muito importante, com destaque para a questão da iniciativa do professor em interagir com os alunos pela rede, onde $80 \%$ dos alunos consideraram muito importante. Este resultado denota a necessidade de maior interação por parte dos alunos com o professor, o que se confirma nas demais questões deste grupo, pois os alunos que consideraram muito importante a interação com o professor neste espaço foram $76 \%$ do total, sendo que a interação com os outros alunos obteve um percentual menor de registros: $72 \%$ do total de respondentes.

Ao analisar tanto a perspectiva de comunicação quanto o processo de interação percebe-se que em todas as questões mais de $95 \%$ dos alunos consideraram importante ou muito importante o uso da rede social virtual na disciplina ministrada. Este resultado demonstra que esta iniciativa foi bem aceita pelos alunos, que tiveram ainda a oportunidade de registrar comentários sobre o processo. 


\subsection{Análise Qualitativa quanto à utilização do Facebook}

De acordo com o objetivo traçado para este estudo, buscou-se conhecer a percepção dos alunos quanto à importância da comunicação e da interação possibilitadas através do grupo virtual no Facebook. Na análise, o foco se deu nas principais falas que apresentassem relações com estes dois quesitos, fosse de forma positiva ou mesmo negativa. Contudo, não foi possível se deparar com avaliações negativas sobre estes fatores, tendo sido recorrentes os comentários positivos sobre a melhoria dos processos de comunicação e interação propiciados pela rede social virtual, conforme pode ser percebido por meio das falas apresentadas no Quadro 1 abaixo:

\begin{tabular}{|c|c|c|}
\hline $\begin{array}{l}\text { Categorias } \\
\text { de Análise }\end{array}$ & $\mathbf{N}^{\mathbf{o}}$ & Fragmentos Ilustrativos \\
\hline \multirow{10}{*}{ Comunicação } & \multirow{10}{*}{1} & [...] o grupo virtual facilita a comunicação entre os alunos e professor. [A11] \\
\hline & & $\begin{array}{l}\text { [...] como a rede social é, por muitos, mais acessada que o próprio e-mail é uma plataforma que fica } \\
\text { fácil a comunicação. [A12] }\end{array}$ \\
\hline & & $\begin{array}{l}\text { [...] A utilização do grupo virtual auxiliou muito o andamento da disciplina durante o semestre, } \\
\text { disponibilizando todo o material básico e o completar no facebook, economizando tempo e dinheiro. } \\
\text { [A15] }\end{array}$ \\
\hline & & $\begin{array}{l}\text { [...] facilitou bastante a comunicação do professor para com seus alunos. Gostei e achei mais eficiente } \\
\text { que muitas outras formas de comunicação que já existem. [A18] }\end{array}$ \\
\hline & & $\begin{array}{l}\text { [...] Achei muito interessante da utilização do facebook como meio de comunicação na disciplina. Foi a } \\
\text { primeira vez que vi a ocorrência de tal fato na UFOP. [A21] }\end{array}$ \\
\hline & & $\begin{array}{l}\text { [...] Devido ao fato de hoje as redes sociais estarem presentes no dia a dia de quase todos, portanto a rede } \\
\text { social foi um meio de facilitar o processo de comunicação. [A30] }\end{array}$ \\
\hline & & $\begin{array}{l}\text { [...] } \mathrm{O} \text { uso do grupo virtual trouxe muita facilidade e eficiência na comunicação professor-aluno. } \\
\text { [A34] }\end{array}$ \\
\hline & & $\begin{array}{l}\text { [...] O grupo facilitou a comunicação com o professor e acesso ao material didático. Sugiro que } \\
\text { continue sendo utilizado. [A } 45]\end{array}$ \\
\hline & & [...] Creio que seja uma boa forma do professor manter os alunos informados. [A46] \\
\hline & & [...] tornou a comunicação ágil e dinâmica. Foi bastante produtivo. [A51] \\
\hline \multirow{3}{*}{ Interação } & \multirow{3}{*}{2} & $\begin{array}{l}\text { [...] Acho de suma importância não só para termos contato só com o professor como também ter maior } \\
\text { interação entre os alunos, já que no horário da aula temos pouco tempo para isso. [A16] }\end{array}$ \\
\hline & & $\begin{array}{l}\text { [...] Esse novo formato de oferta do conteúdo que permite o acesso de acordo com a disponibilidade de } \\
\text { tempo do aluno facilitou também a interação aluno/professor. [A17] }\end{array}$ \\
\hline & & [...] sem falar que era possível tirar duvidas e/ou fazer alguma reclamação por este meio. [A30] \\
\hline
\end{tabular}

Quadro 1 - Melhoria dos processos de comunicação e interação na percepção dos alunos

Fonte: Elaborado com base nos dados da pesquisa

O processo de comunicação do professor para com os alunos foi classificado como facilitado pela rede social virtual, tendo se apresentado de forma mais eficiente, ágil e dinâmica, propiciando ainda uma redução do custo e do tempo gasto pelos alunos se comparado ao que gastam ao reproduzir materiais impressos no 'xerox' da universidade. Houve ainda falas afirmando que tal ferramenta se colocou à frente de outras formas já realizadas por professores das quais são de conhecimento dos alunos. Da mesma forma, o processo de interação mostrou-se de grande importância por ir além do ambiente físico da aula. Tal interação permitiu que dúvidas fossem esclarecidas e reclamações recebidas e resolvidas. $\mathrm{Na}$ fala de um dos alunos [A33], pode-se compreender o quanto as interações puderam ser fortalecidas por meio da rede social virtual, quando argumenta que [...] "o grupo virtual ajuda a unir os estudantes de uma turma, já que nem todos mantem um contato diário ou se conhecem".

Além do objetivo buscado neste estudo, outras categorias surgiram a partir da análise. Contudo, tais categorias só fortalecem e reafirmam em que aspectos a comunicação e a interação se mostraram potencializadas a partir da utilização da rede social virtual. A primeira categoria 'Aprendizagem' é, a nosso ver, o principal resultado das melhorias dos processos de comunicação e interação. Conforme apontado pela grande parte dos alunos, a qualidade do aprendizado melhorou a partir dos materiais complementares disponibilizados, o que levou muitos alunos a se interessarem pelos conteúdos complementares. Isso pode ser comprovado pelo professor em função da 
participação dos alunos no grupo virtual, seja a partir de 'curtidas' nas postagens quanto, especialmente, pelos comentários sobre as postagens de conteúdos complementares ao material didático básico da disciplina ministrado em sala de aula.

As categorias 2, 3 e 4 se mostram como razões que levaram à melhoria do aprendizado dos alunos, sendo elas, respectivamente, 'Acesso a Materiais Didáticos Básicos e Complementares', 'Rapidez no Recebimento de Informações' e 'Acompanhamento da Disciplina'. Em relação ao acesso aos materiais, os alunos parecem concordar que a disponibilização de materiais digitais é mais interessante que a disponibilização de materiais impressos para serem reproduzidos. Além disso, o acesso constante ao Facebook permite que eles fiquem cientes com muita rapidez e praticidade quando novos materiais eram inseridos no grupo virtual. Já em relação à rapidez do recebimento destas informações, os alunos apontam que utilização do Facebook por eles é praticamente diária e em várias partes do dia, o que permite o rápido acesso aos conteúdos disponibilizados em um ambiente que já é utilizado para outros fins. $\mathrm{Na}$ próxima categoria, 'acompanhamento da disciplina', os alunos demonstraram que o acesso contínuo a informações de conteúdo de ensino e de planilhas de notas permitem se inteirar melhor ao longo da disciplina, já que por vezes tais informações nem são buscadas, mas veem de encontro a eles quando estão utilizando a rede virtual para outros fins. Este fato é claramente compreendido se analisada a fala de um aluno (A02) que informa que [...] "com o advento das redes sociais, não vislumbro um melhor ambiente para integrar o estudo, diversão e tecnologia".

Na quinta e última categoria, "Avaliação da Ferramenta de Apoio ao Ensino", buscou-se a percepção dos alunos quando à avaliação geral da utilização do grupo virtual no Facebook por parte do Professor. De modo geral, os alunos mostraram surpresa e contentamento em relação à ferramenta utilizada. Falas como as apresentadas no quadro mostram que, na avaliação dos alunos, a ferramenta se foi positivamente avaliada como um método inovador, como abordagem muito interessante e uma ideia excelente, o que foi avaliado como uma iniciativa válida para tratar de assuntos da disciplina fora da sala de aula.

\section{Considerações Finais}

O presente estudo buscou apresentar os resultados da utilização de um grupo virtual no Facebook como forma complementar para a comunicação e interação de professor e alunos de uma disciplina ministrada na Universidade Federal de Ouro Preto (UFOP). De modo geral, o que se percebe é uma avaliação positiva por parte dos alunos quanto à utilização desta ferramenta ou ambiente de relações/interações. As inovações realizadas na forma de desenvolver a disciplina em questão, a partir de uma mudança/implementação nos processos de comunicação e interação, possibilitou uma melhoria no aprendizado dos alunos, em especial pela disponibilização de materiais complementares aos materiais básicos da disciplina, tais como vídeos e sites.

O estudo colabora, em termos empíricos, para o reconhecimento da importância de se utilizar da tecnologia e do ciberespaço a favor da educação, em especial na utilização de redes sociais virtuais abertas como forma complementar às salas de aula, o que se diferencia em muito das plataformas virtuais de ensino mais clássicas e populares, como no caso do Moodle. Em termos teóricos, este estudo levanta o questionamento e desafios de discussão de metodologias e atividades virtuais como forma de complementar às atividades realizadas em sala de aula, colocando em discussão abordagens inovadoras de um lado e as clássicas de outro, mas de forma a se complementarem e não se anularem no campo da educação. 
As limitações deste trabalho se relacionam com o fato de tal estudo ter sido realizado com uma amostra e não com a população dos alunos da disciplina. É possível que justamente os alunos mais interessados na rede social, na sua utilização e em seus benefícios foram os mais propensos e disponíveis a responderem ao questionário que deu base para este estudo. Contudo, isso é apenas uma suposição, o que não retira a importância das observações das percepções dos alunos quanto à importância das redes sociais virtuais como ferramentas alternativas ao ensino.

A discussão apresentada a partir dos dados primários coletados dá um norte sobre uma forma de se compreender as percepções dos alunos envolvidos no processo de ensino discutido, mas novas pesquisas e estudos podem buscar elucidar de forma mais clara os impactos das redes sociais virtuais nas práticas dos alunos e não apenas na predominância das ações realizadas por professores. Desta forma, outros estudos podem propiciar uma visão mais clara sobre os impactos das relações virtuais de alunos e professores nas suas relações reais, bem como no sentido reverso. Além disso, sugere-se que novos estudos busquem comparar as ferramentas disponibilizadas por redes sociais virtuais em comparação com ferramentas próprias desenvolvidas para o ensino, tal como o Moodle e demais plataformas de ensino à distância no que tange à complementariedade da modalidade à distância para o ensino presencial.

\section{Referências Bibliográficas}

AGUIAR, S. Redes sociais na internet: desafios à pesquisa. In: XXX Congresso

Brasileiro de Ciências da Comunicação. 2007. Anais. Santos: Intercom, 2007.

Disponível em:

<http://www.sitedaescola.com/downloads/portal_aluno/Maio/Redes\%20sociais\%20na\% 20internet-\%20desafios\%20\%E0\%20pesquisa.pdf> Acesso em: 12 jan. 2012.

BOYD, D. The significance of social software. In: BURG, T. N.; SCHMIDT, J. (Eds.) BlogTalks reloaded: social software research \& cases. Norderstedt: Books on demand, 2007. p.15-30. Disponível em: 〈http://www.danah.org/papers/BlogTalkReloaded.pdf> Acesso em: 27 fev. 2012.

CASTELLS, Manuel. A sociedade em rede. São Paulo: Paz e Terra, 1999.

CHEUNG, C.; CHIU, P-Y.; LEE, M. Online social networks: Why do students use Facebook? Computers in Human Behaviour, 27. 2011. Disponível em:

<http://www.sciencedirect.com/science/article/pii/S0747563210002244> Acesso em: 21 out. 2013.

CRUZ, J. A.; QUANDT, C. O. Redes, cooperação e desenvolvimento: estudo de caso em uma rede de associações de coletores de materiais recicláveis. In: ENCONTRO DA ASSOCIAÇÃO NACIONAL DOS PROGRAMAS DE PÓS-GRADUAÇÃO EM ADMINISTRAÇÃO, 31., 2007, Rio de Janeiro. Anais ... Rio de Janeiro: ANPAD, 2007.

LEMOS, A.; LÉVY, P. O Futuro da Internet: em direção a uma ciberdemocracia planetária. São Paulo: Editora Paulus, 2010. 258p.

LÉVY, P. Cibercultura. Trad. Carlos Irineu da Costa. 2.ed. São Paulo: Editora 34, 2000. 260p.

LLORENS, F.; CAPDEFERRO, N. Posibilidades de la plataforma Facebook para el aprendizaje colaborativo en línea. Revista de Universidad y Sociedad del

Conocimiento. 8(2) 31-45. 2011. Disponível em:

<http://rusc.uoc.edu/ojs/index.php/rusc/article/view/v8n2-llorens-capdeferro/v8n2lorens-capdeferro> Acesso em: 21 out. 2013.

MACHADO, J. R.; TIJIBOY, A. V. Redes Sociais Virtuais: um espaço para efetivação da aprendizagem cooperativa. Novas Tecnologias na Educação. CINTED-UFRGS. 
Porto Alegre, v.3, n.1, mai., 2005. Disponível em: <http://seer.ufrgs.br/renote/article/view/13798/7994> Acesso em: 25 jan. 2012. MARTELETO, Regina Maria. Análise das redes sociais: aplicação nos estudos de transferência da informação. Ciência da Informação, Brasília, v. 30, n. 1, p.71-81, jan./abr. 2001. Disponível em: 〈http://www.scielo.br/pdf/\%0D/ci/v30n1/a09v30n1.pdf> Acesso: 18 jan. 2012.

MINHOTO, P. M. L.V. A utilização do Facebook como suporte à aprendizagem da biologia: estudo de caso numa turma do $12^{\circ}$ ano. Dissertação de Mestrado. Escola Superior de Educação. Instituto Politécnico de Bragança. 2012.

PRIMO, A. F. T. A emergência das comunidades virtuais. In: Intercom 1997 - XX Congresso Brasileiro de Ciências da Comunicação, 1997, Santos. Anais... Santos, 1997. Disponível em: <

http://www.sitedaescola.com/downloads/portal_aluno/Maio/A\%20emerg\%EAncia\%20 das\%20comunidades\%20virtuais.pdf >. Acesso em: 18 jan. 2012.

RHEINGOLD, H. Comunidade virtual. Trad. Helder Aranha. Lisboa: Gradiva, 1996. SELWYN, N. Em defesa da diferença digital: uma abordagem crítica sobre os desafios curriculares da Web 2.0. In: DIAS, P.; OSÓRIO, A. (orgs.) Aprendizagem (In)Formal na Web social. Centro de Competências da Universidade do Minho. Braga. 2011. SILVA, L.; GOEL, L.; MOUSADIVIN, E. Exploring the dynamics of blog communities: the case of MetaFilter. Information Systems Journal, v. 19, p. 55-81, 2008.

TAVARES, W.; PAULA, A. P. P. Movimentos Sociais em Redes Sociais Virtuais: Possibilidades de Organização de Ações Coletivas e Ativistas no Ciberespaço. In: ENCONTRO DA ASSOCIAÇÃO NACIONAL DOS PROGRAMAS DE PÓSGRADUAÇÃO EM ADMINISTRAÇÃO, 37., 2013, Rio de Janeiro. Anais ... Rio de Janeiro: ANPAD, 2013. p.1 - 16.

WELLMAN, B.; BERKOWITZ, S. D. Social structures: a network approach. New York: Cambridge University Press, 1988.

WELLMAN, B. Little Boxes, Glocalization, and Networked Individualism. In TANABE, M., BESSELAAR, P. V. D.; ISHIDA, T. (Eds.) Digital Cities II:

Computational and Sociological Approaches. Berlin: Springer-Verlag, 2002. p. 11-25. Disponível em: <http://homes.chass.utoronto.ca/ wellman/publications/littleboxes/littlebox.PDF> Acesso em: 10 fev. 2012. 